\title{
Redefining robot based technologies for elderly people assistance: a survey
}

\author{
Luigi Pagliarini $^{1,2}$ Henrik Hautop Lund ${ }^{1}$ \\ ${ }^{1}$ Center for Playware, Technical University of Denmark, Building 326, 2800 Kgs. Lyngby, Denmark \\ ${ }^{2}$ Academy of Fine Arts of Macerata, Via Berardi, 6, 405111 Macerata, Italy \\ E-mail: luigipagliarini@gmail.com \\ www.playware.elektro.dtu.dk
}

\begin{abstract}
We analyse the state of the art of hi-tech and robot based technologies in terms of Assistive Technology for all patients and, in particular, elderly people assistance and everyday activities aid. We focus on different aspects and characteristics of these tools, such as playfulness, invasiveness, learning-speed, efficiency, short and long-term effect, active vs. passive, etc. We do so by showing the most important existing examples, and by taking into account all the possible factors that might help researchers when thinking of developing appropriate technologies for elderly care, as well as, for their relative assistance personnel. Indeed, while in rehabilitation robotics, a major role is played by the human-machine interface (HMI) used to gather the patient's intent from biological signals, and convert them into control signals for the robotic artefacts, surprisingly, decades of research have not yet declared what the optimal HMI is in this context [1]. Further, there is an urgent need to clarify how various technologies can be a goal or an approach for preventive, rehabilitative and assistive interaction. Therefore, we try to make a first step towards a redefinition of Robotics Assistive Technology.

Keywords: Assistive Technology, Robotics, Prevention, Rehabilitation, Healing, Production.
\end{abstract}

\section{Introduction}

Quite recently, a new field has emerged from both private and public research that has been denominated Assistive Technology. In few cases such research reaches the final state, and releases specific Assistive Technology Products. As matter of fact, Assistive Technology seems to be a generic definition [2] that aims at covering technologies, services, apparatus, products as well as systems mostly used by disadvantaged people, like disabled and elderly ones. The aim is to increase those people's quality of life by helping them reaching the best possible autonomy either in social or private life. In other words, it means giving them the chance to recuperate their impaired or weakened skills, both motor and cognitive ones so that they can execute tasks, which might have become too difficult or too dangerous to them. Therefore, the definition of assistive technologies relies on the classification of either social or medical models of disability. This is an important point that tends to distinguish ludic productions from assistive technologies themselves. Although, this is surely a good way to look at Assistive Technology, along this paper we will try to enrich such a vision by including in the set of Assistive Technology Products tools that might work at a prevention level, a level that from our point of view is still one of the most desirable ones.

Normally, the definition of assistive technology products, as described by Hersh [3], can be classified in at least five "flexible" categories, such as: 
- Mainstream Products (i.e. general population products that are available everywhere);

- Universal Design Products (i.e. products that are conceived to be used by the largest possible group of users) ;

- Assistive Products (i.e. designed to remove barriers for elderly and disabled people);

- Rehabilitation Products (i.e. products designed to restore functions in elderly and disable people);

- Medical Products (i.e. products designed to support a range of health care practices and promote healing).

It is to be noticed that the difference between assistive and rehabilitative products stand in the fact that the assistive products modify the people interaction with the environment by removing the "obstacles" in some way, while the rehabilitative ones are meant to directly re-enable persons, in order to overcome their "limitations".

Once such a product is released, there are a number of different ways of classifying evaluation and evaluation methods (e.g. evaluation can be purely evaluative or diagnostic). By generalising a categorisation inherited from human computer interaction [4], assistive products gives at least four classes:

- Analytical methods: specialists manipulate system models to predict performance.

- User reports: users work with representations or their memories of particular products. (i.e. interviews and questionnaires, etc.).

- Specialist reports: human factors specialists or other professionals assess real products, prototypes or simulations.

- Observational methods: real users assess real products, prototypes or simulations.

Considering the pragmatic of productions, it has been proven that there are factors that are most important in product success and product screening decisions, as well as factors that influence decisions on whether or not to discontinue a product. They are somehow crucial in understanding the effective value of a product and they include $[5,6,7,8]$ :

- Product advantages and expected market reaction;

- Expected financial potential, including market share, sales and market growth and expected profitability;

- The implications for the firm's financial, physical and human resources;

- Fit to current business, organisation, technology, marketing and managerial skills;

- The potential or availability of new products and alternative opportunities, as well as the product range policy.

In this perspective, we will here report some examples of Assistive Technology Products based on Robotics and will compare them to both review the state of the art in such fields' intersection, and to try to underline how it fits with the general standards of Assistive Technology.

\section{Assistive Technology - Examples}

The first examples of Assistive Technology Product we present are the HONDA Walking Assist Device and its Cyberdyne precursor HAL (Fig. 1). They both represent the classical Assistive Technology devices designed to remove barriers for elderly and disabled people, and that help users to support bodyweight to reduce the load on the user's legs while walking, going up and down stairs, and in a semi-crouching position.
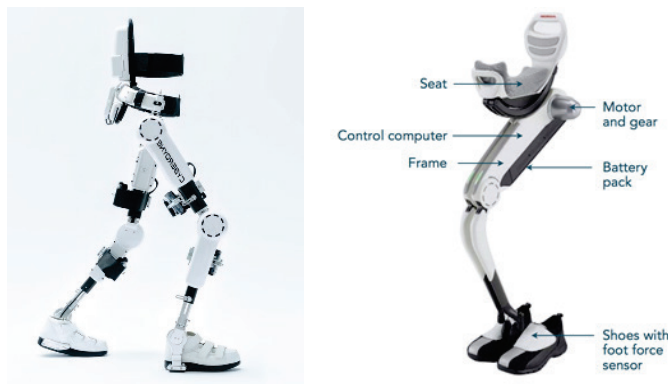

Fig. 1. Cyberdyne HAL, and HONDA's Walking Assist Device. 
Both HAL and Walking Assist are examples of an efficient production that is professionally evaluated throughout specialist reports. They both fit their firm's current business, organisation, technology, marketing and managerial skills and financial potential, including a big potential market share. In other words, in their assistive niche, they represent the perfect product and product design, although they almost exclusively act in the no-rehabilitation zone.

On the same line are the Assistive Robotic Manipulator, as InMotion's Arm, Wrist, Hand and Exoskeletal Arm Robot produced by the Interactive Motion Technologies. These technologies should help patients with Stroke, Cerebral Palsy, Traumatic Brain Injury, Spinal Cord Injuries, Multiple Sclerosis, and Parkinsons to add a level of independence to their lives. These products are evaluated either by the patients and the specialists are well tested and seem to be promising for Assistive Technology Clinical practice, at least.

Further, there are a long number of productions oriented towards becoming caregiver robots. Amongst them, the RIBA (or Robear) developed by researchers at RIKEN and Tokai Rubber Industries (TRI) is the most advanced example. It is in between an Assistive and a Medical Product since besides removing barriers, it is meant to take care of patients, substituting some nursery functions.

Besides those, there are other fields of research, like for example Socially Assistive Robotics - see "The Robotics Primer" [9] for further information - which are currently trying to develop robot-assisted therapies for children with autism spectrum disorders, stroke and traumatic brain injury, and individuals with Alzheimer's Disease and other forms of dementia. But, despite of the fact that the field seems to be promising and important, and with some good indications from the Paro robot by Shibata, there is not an effective Assistive Products outcome, yet.

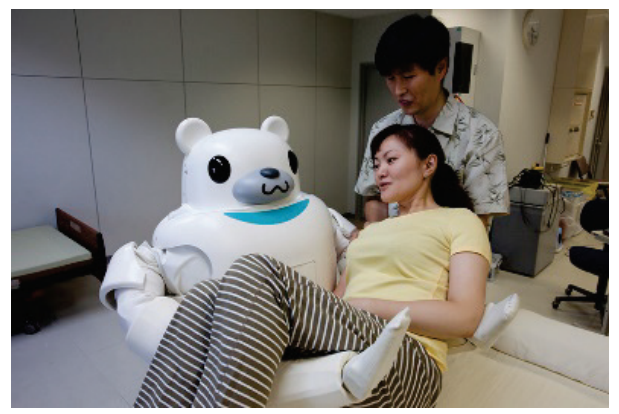

Fig. 2. The RIBA Robot lifting a patient.

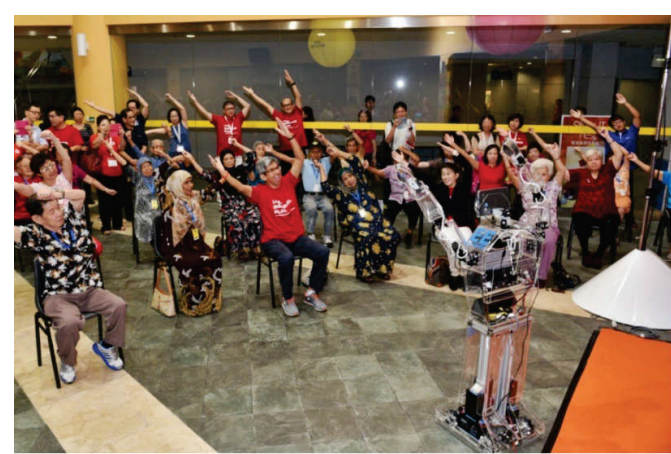

Fig. 3. RoboCoach.

On the contrary, on the side of what we will here call Active Assistive Robotics the production can be considered much more concrete. One example could be

RoboCoach, developed by Yaacob Ibrahim at the Ngee Ann Polytechnic, that coaches the elderly in performing 15 types of arm exercise each week, recognizing a human voice that tells it to start the workout.

Vice versa, on the Rehabilitative Robotics Products side there seems to be a quite fruitful production that results in an enlarging number of companies specialized on different branches of artefacts. AlterG Inc., that makes anti-gravity treadmills and bionic legs for athletic training and physical therapy. Ekso Bionics, that makes robotic exoskeletons providing gait training and neurorehabilitation by extending the individual's strength and also helping the therapist to work with their patients. Hocoma, that provides spine evaluation devices that enables computer-assisted analysis and display of the spine's shape and mobility, or robotic gait orthosis in which the patient's legs are guided to a preprogrammed physiological gait pattern on the treadmil. Bioextreme, specialized in robotics rehabilitation innovative Error Enhancement treatment for patients. Intead Technologies and Kinestesica, specialized in neuromotoric disorders. A very interesting approach is the one from the Kinetic Muscles that is merging digital gaming, robotic, and telemedicine technologies with the most up-to-date discoveries in neurorehabilitation science to provide solutions that are practical and clinically effective for engaging post-stroke rehabilitation. MediTouch, that produces wearable motion capture devices and dedicated rehabilitation software that allow patients with upper and/or lower extremity movement dysfunction to practice intensive virtual functional task training. SynTouch that makes the only sensor technology in the world that endows robots 
with the ability to replicate the human sense of touch, or TouchBionics provider of world-leading prosthetic technologies and supporting services. Besides them, the technologies realized by Kinova, Rehab-Robotics, RehaStim, YouRehab and the above mentioned Interactive Motion Technologies are very interesting.

All of them work with patients suffering from partial spinal cord injuries, strokes, multiple sclerosis, traumatic brain injuries, cerebral palsy, Parkinson's disease: generally speaking with nervous system injuries and patients with minimal forearm strength and problems in standing and walking. Some of these have a mainly passive approach while other push a proactive exercising. Amongst these last ones a few are also targeting playfulness as a philosophy. They also differ as level of Human-Machine Interface (HMI) invasiveness, although in different cases appropriate and engaging HMI design to be the only effective way.

On the Robotics Medical Products side, there is a large production. Medical apparatus, especially in surgery, are day-by-day moving towards a roboticsbased practice. As, for example, Magnetic Microbots, a group of tiny robots used in various operations, such as removing plaque from a patient's arteries or helping with ocular conditions and disease screenings. In short, robotic advancements are used to better the day-to-day lives of patients, helping them eat (e.g Bestic Arm), or helping a patient regain her ability to walk (e.g Toyota's Healthcare Assistants). Obviously, robotics is a very attractive industry since within the hospitals, a robot's work can fulfil the full-time work of one or more employees (e.g Aethon TUG) and outside of them caregivers use robots to enhance telemedicine and care for those restricted to their homes (e.g. Vasteras Giraff) or, surprisingly enough, in the open (e.g. Anybots). Some of them are playful and non-invasive ones, as those used to enhance the therapy of developmentally disabled children between 5 and 12 years old (e.g. CosmoBot).

On the Mainstream and Universal Design Products side there is a large number of contributions which mainly focus their attention on fitting products in the ecological system of humans. Therefore the products try to be portable, fit in the apartment or outside homes trying to follow the scheme of the "walk up and use" design philosophy, to be scalable, to allow user to initiate interaction, to provide different choices for any given task, to provide multiple and multimodal output and feedback, to provide mutable functionality and to target the largest number of people and contexts. See [10] for further information.

Good examples can be considered the iRobot products, the Toyota's i-REAL, the Universal Robots products, and many other products for the toys and gaming industry (e.g. WowWee) or, sometimes, those that are specifically targeted for the assistive technology market (e.g. Cyberdyne).

\section{Moto tiles}

In this context, we present our Moto tiles, robot tiles that light up when you touch them and that are mostly used to improve the balance of the elderly, and strengthen their muscles and endurance with a high grade of efficiency, as it has been demonstrated by scientific tests [11, 12]. Despite of their specific Medical Technology target and rehabilitative application, Moto tiles use can be easily translated in quite different contexts since they fulfil almost all of the typical criteria of the Universal Design Products. Their use is easy to learn. They are multimodal and can easily adapt to any kind situation, either indoor or outdoor, since they are very portable, reconfigurable and well fit to any context and user's age. Therefore, they might be used either as gaming or educational platform. More than that, they show their best when combining playfulness with fitness, rehab or educational exercises, all together. Besides, if compared with the mainstream assistive technology or medical technology products, they show their peculiarity in being a mostly "active" tool. Indeed, the software platform they use promotes users' motor and/or cognitive activation at the best and tends to adapt to the rate of users' responses in a fairly adaptive way.

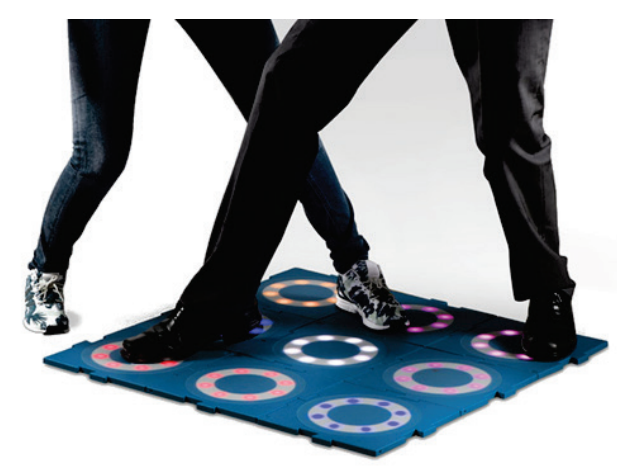

Fig. 4. Moto tiles - an example of an active tool for prevention and rehabilitation (www.mototiles.com). 
Furthermore, they represent a rather unique example of Assistive Technology Robotics applied to "prevention", a "medical zone" which is rarely considered within the field and that, on the contrary, might represent one of the most important fields of robotics applications, in the future.

In other words, the Moto tiles might represent, by themselves, a new dimension of how to apply robotics within an Assistive Technology context, by promoting the idea of cure as "prevention" and therefore by stressing the "activation" at its best. The Moto tiles also show how, by focusing on such a direction, Assistive Technology can reach important results in either short or long term effects.

\section{Discussion and Conclusion}

In this paper we discussed the most important general principles of Assistive Technologies and, in particular, analysed the state of the art Robotics Assistive Technologies. At a first sight, it seems that much is to be done and that despite of decades of research, both the optimal HMI is in this context is still to be understood and the borders of such a field is to be defined. We also reported the most successful examples of market and science industries trying to underline differences amongst what is to be considered a good or a bad example of production. By doing that we also highlighted the different application fields and how production itself is moving within them, by providing possible solutions under different circumstances.

In this context, we presented the Moto tiles robotic system as an innovative example on how to approach Assistive Technology, by opening new fields of application and new approaches to users' attitude. Indeed, in our opinion, it is important to continuously change the philosophy by which we approach robotics (i.e. either as research and as an applicative field of production), trying to find new technological and behavioural scenarios that better fit to users' need of easily understanding and use of technology in assistive, rehabilitative, and preventive robotics. We believe that, ideally, as already happened in telecommunication technologies, user and patient oriented robotic technologies must get simpler, adaptable, and enlarge the range of action as much as possible.

\section{References}

1. http://journal.frontiersin.org/article/10.3389/fnbot.2014.0 0024/full?utm_source=newsletter\&utm_medium $=$ email\& utm_campaign=Robotics_and_AI-w49-2015

2. M.A. Hersh, and M.A. Johnson. Assistive technology for visually impaired and blind people. (Springer Verlag. 978-1-84628-866-1. 2008)

3. M.A. Hersh. 2010. The Design and Evaluation of Assistive Technology Products and Devices Part 2: Evaluation of Assistive Products. In: JH Stone, M Blouin, editors. International Encyclopedia of Rehabilitation.

4. A. Whitefield, Wilson F, Dowell J. 1991. A framework for human factors evaluation. Behaviour and Information Technology 10(1):65-79.

5. G. J. Avlonitis 1993. Project Dropstrat: what factors do managers consider in deciding whether to drop a project. European Journal of Marketing 27(4):35-57.

6. Brentani de U. 1986. Do firms need a custom-designed new product screening model. Journal of Product Innovation Management 3:108-119.

7. J. R. Schmidt, Sarangee, KR, Montoya MM. 2009. Exploring new product development project review practices. Journal of Product Innovation Management 26:520-535

8. X. M. Song, Parry ME. 1996. What separates Japanese new product winners from losers. Journal of Product Innovation Management 13:422-439.

9. https://mitpress.mit.edu/books/robotics-primer

10. http://repository.cmu.edu/cgi/viewcontent.cgi?article $=10$ $44 \&$ context $=$ hcii

11. H. H. Lund, Modular Robotics for Playful Physiotherapy, in Proceedings of IEEE International Conference on Rehabilitation Robotics (IEEE Press, 571-575, 2009)

12. H. H. Lund, and J. D. Jessen, Effects of short-term training of community-dwelling elderly with modular interactive tiles. GAMES FOR HEALTH: Research, Development, and Clinical Applications, 3(5), 277-283, 2014. 